\title{
EFECTO DEL EXTRACTO DEL FRUTO DE Solanum melongena "BERENJENA" EN CONEJOS HIPERCOLESTEROLÉMICOS
}

Lidia Cruz Neyra ${ }^{1}$

\begin{abstract}
RESUMEN
El propósito de la presente investigación fue determinar el efecto del extracto crudo del fruto de la «berenjena», Solanum melongena, sobre el perfil lipídico y la peroxidación lipídica en conejos hipercolesterolémicos. Se utilizaron treinta conejos, separados en tres grupos que recibieron dieta control (GC), dieta hipercolesterolémica (GH) y dieta hipercolesterolémica y extracto de berenjena (GB). Se evaluaron el perfil lipídico y la peroxidación en la lipoproteína de baja densidad (LDL) nativa y oxidada. El colesterol plasmático, LDL y triglicéridos que se incrementaron en el grupo GH se redujeron en 19, 29 y 38\% respectivamente en el grupo GB. En el grupo GB disminuyeron el contenido de malonaldehído (MDA) en las LDL nativas (56\%) y oxidadas (22\%) en comparación al grupo GH (p<0.05). Se evidencia que la berenjena tiene un papel protector sobre la peroxidación lipídica.
\end{abstract}

Palabras claves: Berenjena, hipercolesterolemia, peroxidación lipídica

\section{SUMMARY}

The purpose of this study was to determine the effect of crude extract from the fruit of the eggplant, Solanum melongena, on the lipid profile and lipid peroxidation in hypercholesterolemic rabbits. Thirty rabbits were used, separated into three groups that received control diet (GC), hypercholesterolemic diet (GH) and hypercholesterolemic diet and extract eggplant (GB). These were evaluated lipid profile and lipid peroxidation in low-density lipoprotein (LDL) native and oxidized. The plasma cholesterol, LDL and triglycerides are increased in the GH group, but were reduced by 19, 29 and 38\% respectively in group GB. The group GB decreased the content of malonaldehide (MDA) in the native LDL (56\%) and oxidized (22\%) compared to the GH group (p $<0.05)$. It was evident that the eggplant has a protective role on lipid peroxidation.

Key words: Eggplant, hypercholesterolemia, lipid peroxidation

\section{INTRODUCCIÓN}

En la actualidad existe un consenso en relación al papel del estrés oxidativo en la aterosclerosis, que representa un mayor estado de oxidación de lípidos y proteínas en la pared vascular. (Witztum, 1994, Stocker et al. 2004)

La hipótesis de la modificación oxidativa en la aterosclerosis predice que la oxidación de las lipoproteínas de baja densidad (LDL) es un evento temprano en la aterosclerosis y su modificación oxidada contribuye a la formación de ateromas. Esta hipótesis, se evidencia in vitro cuando LDL oxidada forma células espumosas (foam cell) e in vivo tiene una serie de actividades potencialmente proaterogenica, y varias de ellas estructuralmente no vinculadas a la inhibición por antioxidantes en aterosclerosis experimental en animales. Un consenso en la enfermedad vascular destaca la importancia de los eventos oxidativos, además de la oxidación del LDL (Aviram, 1996).

Los eventos oxidativos incluyen la producción de especies reactivas de oxígeno y nitrógeno por las células vasculares, así como las modificaciones oxidativas que contribuyen a importantes manifestaciones clínicas de las enfermedades cardio-

\footnotetext{
${ }^{1}$ Laboratorio de Bioquímica y Nutrición. Facultad de Ciencias Biológicas. Universidad Ricardo Palma.

E-mail: lcruzne@hotmail.com
} 
vasculares, tales como la disfunción endotelial y la interrupción de la placa. A pesar de estos abundantes datos, sin embargo, siguen habiendo problemas fundamentales con la implicancia de la modificación oxidativa como un requisito patofisiologicamente importante de la aterosclerosis (Ylä-Herttuala, 1991). El daño oxidativo que se produce por el desequilibrio entre fenómenos antioxidantes/proxidantes parece crucial en el origen de la ateroesclerosis, y esto aumenta la posibilidad que los antioxidantes, como la vitamina $\mathrm{C}$, betacaroteno y en especial el alfa tocoferol (vitamina E), puedan prevenir o retardar el desarrollo de esta enfermedad (Miller, 1998, Stocker y Keaney, 2004)

Por otro lado, hay una corriente de utilizar dietas ricas en alimentos que contengan antioxidantes como es el caso del uso de Solanum melongena L "berenjena", por lo que se deberá estudiar sus principios químicos que tengan una mayor implicancia en el secuestro de radicales libres.

La berenjena es una especie vegetal originaria del sudeste asiático, es consumida cocida o cruda. Estudios farmacológicos demuestran propiedades antiinflamatorias, así como efecto antioxidante de preparados del fruto dada la alta composición de flavonoides. Se ha reportado 115 compuestos de diversa familias química, los que se encuentran en el fruto y corresponden a aminoácidos (alanina, 5hidroxitriptamina, arginina, glicina, leucina, serina), ácidos carboxílicos (alfa-linolénico, araquidónico, ascórbico, aspártico, glutámico, oxálico, palmítico), aminas (fenilalanina, triptamina), alcaloides (isoescopoletina, solanina, solanidina), flavonoides (delphinidin-3-rutinósido-3-(4'-coumaroilrutinósido)-5glucósido) y oligoelementos (aluminio, bario, boro, cadmio, calcio, cobre, hierro, magnesio, potasio, selenio, sodio).

Sudheesh et al., 1999 reportaron que flavonoides aislados de Solanum melongena (berenjena) muestra una potente actividad antioxidante en ratas alimentadas con dieta hipercolesterolémica a quienes se les administró $1 \mathrm{mg}$ de extracto de flavonoide por 100 gramos de peso / día. Las concentraciones de malonaldeído, hidroperóxidos y conjugados dienos fueron sgnificativamente disminuidos. Los niveles de glutationa aumentaron y significativamente estimularon la actividad de catalasa, que podría ser la responsable de los efectos de los flavonoides de la berenjena.

Botelho et al., 2004 evaluaron el efecto de la berenjena en el metabolismo de colesterol y la aterogenesis en ratones LDLR (homocigote para receptor de LDL). Los animales fueron alimentados con dieta normal o hipercolesterolémica por doce semanas, recibiendo además el grupo control agua y el grupo experimental extracto de berenjena. Al término del experimento fueron evaluados el estrés oxidativo a través de la formación de conjugados dienos, anticuerpos anti LDL oxidada por inmunoensayo. El colesterol total y las lipoproteínas aterogénicas no disminuyeron con la ingesta de extracto de berenjena, pero incrementaron los anticuerpos anti LDL oxidada indicando un alto nivel oxidativo . Asimismo el extracto de berenjena no disminuyó el colesterol plasmático, ni la placa aterogénica. Sus resultados no sostienen el uso de extracto de berenjena como agente hipocolesterolémico.

El propósito de la presente investigación fue determinar el efecto del extracto crudo del fruto de la "berenjena" sobre el perfil lipídico y la peroxidación lipídica en conejos hipercolesterolémicos.

\section{MATERIALES Y MÉTODOS}

Se usaron conejos machos de la raza Nueva Zelandia de aproximadamente 2.60 kilos de peso, los cuales fueron colocados en jaulas independientes. Las condiciones ambientales fueron: temperatura promedio $22-25^{\circ} \mathrm{C}$, humedad $75 \%$ y un foto período de 12 horas.

Los animales de experimentación fueron separados en 3 grupos como sigue: Grupo I, dieta control (GC); Grupo II, dieta hipercolesterolémica (GH), conteniendo $0.5 \%$ y $10 \%$ de aceite de coco y Grupo III, dieta hipercolesterolémica $+20 \mathrm{ml}$ de extracto de berenjena (vía nasofaríngea), GB. La dieta fue proporcionada diariamente a razón de 100 gramos y agua ad libitum, durante 30 días, luego de los cuales los animales fueron sacrificados previo ayuno de 14 horas, colectándose la sangre.

Fueron determinados enzimáticamente los valores de triglicéridos (TG) y colesterol total (CT). La LDL se determinó por precipitación selectiva mediante el uso de heparina. Dicho valor se obtiene por diferencia entre los valores de colesterol total y los de VLDL (lipoproteína de muy baja densidad) HDL (lipoproteína de alta densidad). HDL se obtuvo precipitando selectivamente las lipoproteínas LDL y VLDL, y quedando HDL en solución, determinándose el contenido de colesterol, según Trinder 1998.

Para la determinación de la peroxidación lipídica se aisló LDL por ultracentrifugación de acuerdo al procedimiento de Havel et al. 1995. La oxidación de LDL fue realizada mediante incubación de 100 ug de proteína/ mL en buffer fosfato salino $1 \mathrm{mM}$, pH 7.4 en presencia de sulfato de cobre $(5 \mathrm{mM})$ a $37 \mathrm{C}$ por 24 horas. La determinación del contenido de Malondialdehído (MDA) fue realizada según el método de Buege y Aust, 1978, utilizando 150 ug de proteína de LDL. El fundamento es la reacción de 
MDA, producto de lipoperoxidación con el ácido tiobarbitúrico, formándose el complejo MDA-TBA, que se determina a $535 \mathrm{~nm}$ por absorbancia.

El análisis estadístico se realizó usando el test de Kruskal Wallis a un nivel de significancia de $\mathrm{p}<0.05$.

\section{RESULTADOS}

Los valores del perfil lipídico de los tres grupos de conejos: control (GC) hipercolesterolémico (GH) y el tratado con extracto de berenjena (GB) son mostrados en la Tabla 1.

Los valores de colesterol total CT, LDL, VLDL y Triglicéridos aumentaron significativamente en el grupo sometido a dieta hipercolesterolémica, en comparación con el grupo control; mientras que el grupo tratado con extracto de berenjena disminuyeron significativamente. $(\mathrm{p}<0.05)$. Sin embargo, no hay diferencias significativas para los niveles de VLDL y HDL entre los grupos hipercolesterolémicos y tratados con extracto de berenjena.

La concentración de malonaldehído en las LDL nativas y oxidada aumentaron significativamente $(\mathrm{p}<$ 0.05) en los animales del grupo hipercolesterolémicos en relación al grupo control y disminuyeron en el grupo tratado con extracto de berenjena (Tabla 2)

Tabla 1. Perfil Lipídico en conejos según dieta

\begin{tabular}{lccc}
\hline mg/dL & Grupol (Control) & $\begin{array}{c}\text { Grupo II } \\
\text { (Hipercolesterolémicos) }\end{array}$ & $\begin{array}{c}\text { Grupo III } \\
\text { (Hipercolesterolémico } \\
+ \text { extracto de } \\
\text { berenjena) }\end{array}$ \\
\hline CT & $59.60 \pm 5.39^{\mathrm{c}}$ & $1,360 \pm 59.80^{\mathrm{a}}$ & $1,097.70 \pm 80.90^{\mathrm{b}}$ \\
C-VLDL & $9.7 \pm 0,16^{\mathrm{b}}$ & $410.53 \pm 42.50^{\mathrm{a}}$ & $435.89 \pm 67.54^{\mathrm{a}}$ \\
C-LDL & $16.91 \pm 2.40^{\mathrm{c}}$ & $841.33 \pm 140.62^{\mathrm{a}}$ & $595.87 \pm 207.06^{\mathrm{c}}$ \\
C-HDL & $32.85 \pm 5.65^{\mathrm{b}}$ & $49.19 \pm 4.63^{\mathrm{a}}$ & $32.85 \pm 5.15^{\mathrm{a}}$ \\
TG & $119.00 \pm 35.26^{\mathrm{c}}$ & $326.54 \pm 78.61^{\mathrm{a}}$ & $119.00 \pm 35.26^{\mathrm{b}}$ \\
\hline
\end{tabular}

CT= colesterol total; TG= triglicéridos, VLDL= lipoproteína de muy baja densidad, LDL=lipoproteína de baja densidad, HDL= lipoproteína de alta densidad. Letras distintas indican diferencias significativas entre grupos $(p<0.05)$ según el test de Kruskall -Wallis.

Tabla 2. Niveles de peroxidación lipídica en LDL de conejos según dieta

\begin{tabular}{lccc}
\hline \multicolumn{1}{c}{ mg/dL } & Grupol (Control) & $\begin{array}{c}\text { Grupo II } \\
\text { (Hipercolesterolémicos) }\end{array}$ & $\begin{array}{c}\text { Grupo III } \\
\text { (Hipercolesterolémico } \\
\text { + extracto de } \\
\text { berenjena) }\end{array}$ \\
\hline $\begin{array}{l}\text { MDA-LDL } \\
\text { Oxidada } \\
\begin{array}{l}\text { MDA-LDL } \\
\text { nativa }\end{array}\end{array}$ & $31.21 \pm 5.06^{\mathrm{a}}$ & $57.23 \pm 5.26^{\mathrm{a}}$ & $44.67 \pm 7.42 \mathrm{~b}$ \\
\hline
\end{tabular}

LDL=lipoproteína de baja densidad, MDA-LDL nativa y oxidada = nanomoles de malonaldehído $/$ mg de proteína de la fracción de LDL. Letras distintas indican diferencias significativas entre grupos $(p<0.05)$ según el test de Kruskall -Wallis 


\section{DISCUSIÓN}

La administración de dietas enriquecida con colesterol y aceite de coco a conejos de la raza Nueva Zelandia, durante treinta días mostró un incrementó de los niveles de colesterol, y de las lipoproteínas plasmáticas, cuando se compara con los niveles de conejos sometidos a una dieta control.

En los animales hipercolesterolémicos tratados con extracto de berenjena, se observó una reducción del 19\% del colesterol total en comparación con el grupo hipercolesterolémico, esta disminución fue observada por Mitschek en 1975 en tejidos aórticos de conejos hipercolesterolémicos, a través de su estudio histológico cuyas alteraciones fueron muy discretas en aquellos animales tratados con berenjena. Asimismo Cruz et al. 1997 reportó que tratando a pacientes hipercolesterolémicos con extracto de berenjena y jugo de naranja observaron una reducción del colesterol total y de las fracciones LDL y VLDL sin observar cambios en los valores de HDL.

Se ha reportado que la berenjena tiene flavonoides entre ellos destaca el delphin-3-rutinosido-3-(4'coumaroilrutinosido)- 5'glucósido, además de contener más de 115 compuestos. Los flavonoides podrían jugar un rol importante en la actividad antioxidante que evitaría la oxidación de las lipoproteínas, particularmente LDL, a quien se le atribuye papel importante en la formación de la placa aterogénica.

De manera que el efecto antioxidante podría ser una causa de la observación de la resistencia de LDL a oxidarse como se aprecia en la tabla 2; sin embargo, se necesita realizar mayores pruebas para establecer las propiedades de la berenjena como reductor de la oxidación y disminución de las concentraciones de colesterol total y de las lipoproteínas plasmáticas.

\section{LITERATURA CITADA}

AVIRAM, M. 1996. Interaction of oxidized low density lipoprotein with macrophages in atherosclerosis, and the antiatherogenicity of antioxidants. Eur. J. Clin. Chem. Clin. Biochem, 34(8):599-608.
BOTELHO, F. V. , Enéas, L. R, Cesar G.C., Bizzotto, C.S., Tavares, E., Oliveira, F.A, Gloria, M.B., Silvestre, M., Arantes, E. y Alvarez-Leite, J. 2004. Effects of eggplant (Solanum melongena) on the atherogenesis and oxidative stress in LDL receptor knock out mice (LDLR). Food and Chemical Toxicology, 42 (8):. 259-267.

BUEG, J.A; Aust. S.D. 1978. Microsomal Lipid peroxidation. Methods of Enzimology., 52: 302310. New York.

CRUZ, J., Teixeira, J.E. 1997. Tratamento das dislipidemias de hipertensos essenciais com berinjela (solanum melongena). Revista Soc. Cardiology ESP. v.7 suppl: 19.

HAVEL, R.J.; Bragdon, J.H. 1995. The distribution and chemical composition of ultracentrifugally separated lipoproteins in human serum. Journal Clinical Investigation. 34: 1345-53.

MILLER III ER, Appel LJ, Risby TH. 1998. Effect of dietary patterns on measures of lipid peroxidation. Circulation, 98:2390-2395.

MITSCHEK, Gh. 1975. Effect of solanum melongena on experimental atheromatosis. Histological studies on cholesterol induced atheromatosis in rabbit in mean and long term test. Experimental Pathology, 10. P: 156-166.

STOCKER R. y Keaney, J.F. 2004. Role of Oxidative Modifications in Atherosclerosisthe . American Physiological Society. Rev 84:1381-1478,

SUDHEESH ,S., Sandhya, C., Asha Sarah Koshy y Vijayalakshmi, N. R. 1999. Antioxidant activity of flavonoids from Solanum melongena .Phytotherapy Research, 13 (5):.393-396

WITZTUM JL. 1994. The oxidation hypothesis of atherosclerosis. Lancet 344:793-795.

YLÄ-HERTTUALA, S. (1991) Macrophages and oxidized low density lipoproteins in the pathogenesis of atherosclerosis. Ann. Med., 23(5):561-567. 